\title{
Travelling Wave Solutions for Two Generalized Hirota-Satsuma Coupled KdV Systems
}

\author{
Engui Fan \\ Institute of Mathematics, Fudan University, Shanghai 200433, P.R. China. \\ Reprint requests to Dr. E. F.; Fax: 86-21-65643045, E-mail: faneg@fudan.edu.cn
}

Z. Naturforsch. 56a, 312-318 (2001); received January 3, 2001

In this paper we present an extended tanh method that utilizes symbolic computations to obtain more travelling wave solutions for two generalized Hirota-Satsuma coupled KdV systems in a unified way. The key idea of this method is to take full advantage of a Riccati equation involving a parameter and use its solutions to replace the tanh-function by the tanh method. It is quite interesting that the numbers and types of the travelling wave solutions can be judged from the sign of the parameter.

Key words: Generalized Hirota-Satsuma Coupled KdV System; Travelling Wave Solution; Symbolic Computation.

In this paper we investigate the two generalized HirotaSatsuma coupled KdV systems

$$
\begin{aligned}
& u_{t}=\frac{1}{4} u_{x x x}+3 u u_{x}+3\left(-v^{2}+w\right)_{x}, \\
& v_{t}=-\frac{1}{2} v_{x x x}-3 u v_{x}, \\
& w_{t}=-\frac{1}{2} w_{x x x}-3 u w_{x}
\end{aligned}
$$

and

$$
\begin{aligned}
u_{t} & =\frac{1}{2} u_{x x x}-3 u u_{x}+3(v w)_{x}, \\
v_{t} & =-v_{x x x}+3 u v_{x}, \\
w_{t} & =-w_{x x x}+3 u w_{x} .
\end{aligned}
$$

The system (1) was proposed by Satsuma and Hirota [1]. They found its 3-soliton solutions and showed that the well-known Hirota-Satsuma equation [2]

$$
\begin{aligned}
& u_{t}=\frac{1}{2} u_{x x x}+3 u u_{x}-6 v v_{x}, \\
& v_{t}=-v_{x x x}-3 u_{x}
\end{aligned}
$$

is a special case of the system (1) with $w=0$ and recalling the transformation $x \rightarrow \sqrt{2} x, t \rightarrow \sqrt{2} t$. Recently, starting from its bilinear form, Tam, Ma and Hu revisited the system (1) and found a new type of soliton solutions [3]. The system (2) is a new generalized Hirota-Satsuma coupled $\mathrm{KdV}$ system proposed by $\mathrm{Wu}$, Geng, and $\mathrm{Hu}$ [4]. By introducing a $4 \times 4$ matrix with three potentials, they derived a new hierarchy of nonlinear evolu- tion equations which exactly contains the system (2). A new complex coupled KdV system and Hirota-Satsuma equation can be deduced from the system (2) as $w=v^{*}$ and $w=v$, respectively. However, to our knowledge no exact solution for the system (2) has been found as yet.

In this work we use a symbolic computation based approach to find more travelling wave solutions for the systems (1) and (2). The key idea of our method is simple and proceeds as follows: For a given partial differential equation, say in two variables,

$$
\mathrm{H}\left(u, u_{x}, u_{t}, u_{x x}, \ldots\right)=0,
$$

we first consider its travelling wave solutions $u(x, t)=$ $U(\xi), \xi=x+\beta t$ to reduce (4) to an ordinary differential equation. The next crucial step is expressing the solution sought for by the form

$$
u(x, t)=U(\xi)=\sum_{i=0}^{m} a_{i} \varphi^{i}
$$

with

$$
\varphi^{\prime}=d+\varphi^{2},
$$

where $\varphi=\varphi(\xi)$. The parameter $m$ can be found by balancing the highest order linear term with the nonlinear terms. Substituting (5) and (6) into the relevant ordinary differential equation will yield a set of algebraic equations with respect to $a_{i}, d, \beta$ because the coefficients of $\varphi^{i}$ have to vanish. From these relations, $a_{i}, d$ and $\beta$ can be determined. It is easy to see that there are some advantages in using the Riccati equation (6) instead of the tanh- 
function in the tanh method [5-7]. First, the Riccati equation (6) admits several solutions:

$$
\begin{aligned}
\varphi= & -\sqrt{-d} \tanh (\sqrt{-d} \xi), \\
& -\sqrt{-d} \operatorname{coth}(\sqrt{-d} \xi), \text { for } d<0, \\
\varphi= & -\frac{1}{\xi}, \text { for } d=0, \\
\varphi= & \sqrt{-d} \tan (\sqrt{-d} \xi), \\
& -\sqrt{-d} \cot (\sqrt{-d} \xi), \text { for } d>0 .
\end{aligned}
$$

This method can produce more travelling wave solutions than the tanh method [5-7]. We can recover the solitary wave solutions that had been found by the tanh method, but for some equations, with no extra effect, we can also pick up new and more general solutions. It is quite interesting that we can use the sign of $d$ in the Riccati equation (6) to judge the numbers and types of such travelling wave solutions. For instance, if $d<0$, we are sure that (4) admits tanh-type and coth-type travelling wave solutions according to (7). Especially (4) will possess five types of travelling wave solutions according to (7)-(9) if $d$ is an arbitrary parameter. The algorithm presented here is also a computerized method in which generating an algebraic system from (4) and solving it are two key procedures, laborious to do by hand. But it is easily implemented on a computer with the help of Mathematica. The outputs of solving the algebraic system by a computer comprise a list of the form $\left\{d, \beta, a_{0} \ldots\right\}$. In general, if $d$ or any of the parameters is left unspecified, it is to be regarded as arbitrary for the solution of (4). For simplicity we write

$$
T=\tanh (\sqrt{-d} \xi), \quad S=\tan (\sqrt{d} \xi),
$$

and coth-type and cot-type solutions are omitted in this paper since they always appear in pairs with tanh-type and tan-type solutions, respectively, according to (7) and (9).

Let us solve the system (1) by the above method. We first make the transformation $u(x, t)=U(\xi), v(x, t)=V$ $(\xi), w(x, t)=W(\xi), \xi=x+\beta t$ and change system (1) into the form

$$
\begin{aligned}
& \beta U^{\prime}=\frac{1}{4} U^{\prime \prime \prime}+3 U U^{\prime}+3\left(-V^{2}+W\right)^{\prime}, \\
& \beta V^{\prime}=-\frac{1}{2} V^{\prime \prime \prime}-3 U V^{\prime} \\
& \beta W^{\prime}=-\frac{1}{2} W^{\prime \prime \prime}-3 U W^{\prime} .
\end{aligned}
$$

Balancing the highest order linear term with nonlinear terms in (10) admits the following ansatz

$$
\begin{aligned}
& u=a_{0}+a_{1} \varphi+a_{2} \varphi^{2}, \\
& v=b_{0}+b_{1} \varphi+b_{2} \varphi^{2}, \\
& w=c_{0}+c_{1} \varphi+c_{2} \varphi^{2} .
\end{aligned}
$$

Substituting (11) into (10) and using Mathematica yields an algebraic system of $a_{i}, b_{i}, c_{i}(i=0,1,2), d$ and $\beta$ :

$$
\begin{aligned}
& -\frac{1}{2} d^{2} a_{1}+d \beta a_{1}-3 d a_{0} a_{1}+6 d b_{0} b_{1}-3 d c_{1}=0, \\
& -3 d a_{1}^{2}-4 d^{2} a_{2}+2 d \beta a_{2}-6 d a_{0} a_{2}+6 d b_{1}^{2} \\
& \quad+12 d b_{0} b_{2}-6 d c_{2}=0, \\
& -2 d a_{1}+\beta a_{1}-3 a_{0} a_{1}-9 d a_{1} a_{2}+6 b_{0} b_{1} \\
& \quad+18 d b_{1} b_{2}-3 c_{1}=0, \\
& -3 a_{1}^{2}-10 d a_{2}+2 \beta a_{2}-6 a_{0} a_{2}-6 d a_{2}^{2}+6 b_{1}^{2} \\
& \quad+12 b_{0} b_{2}+12 d b_{2}^{2}-6 c_{2}=0 \\
& -\frac{3}{2} a_{1}-9 a_{1} a_{2}+18 b_{1} b_{2}=0, \\
& -6 a_{2}-6 a_{2}^{2}+12 b_{2}^{2}=0, \\
& d^{2} b_{1}+d \beta b_{1}+3 d a_{0} b_{1}=0, \\
& 3 d a_{1} b_{1}+8 d^{2} b_{2}+2 d \beta b_{2}+6 d a_{0} b_{2}=0, \\
& 4 d b_{1}+\beta b_{1}+3 a_{0} b_{1}+3 d a_{2} b_{1}+6 a_{1} b_{2}=0, \\
& 3 a_{1} b_{1}+20 d b_{2}+2 \beta b_{2}+6 a_{0} b_{2}+6 d a_{2} b_{2}=0, \\
& 3 b_{1}+3 a_{2} b_{1}+6 a_{1} b_{2}=0, \quad 12 b_{2}+6 a_{2} b_{2}=0, \\
& d^{2} c_{1}+d \beta c_{1}+3 d a_{0} c_{1}=0, \\
& 3 d a_{1} c_{1}+8 d^{2} c_{2}+2 d \beta c_{2}+6 d a_{0} c_{2}=0, \\
& 4 d c_{1}+\beta c_{1}+3 a_{0} c_{1}+3 d a_{2} c_{1}+6 d a_{1} c_{2}=0, \\
& 3 a_{1} c_{1}+20 d c_{2}+2 \beta c_{2}+6 a_{0} c_{2}+6 d a_{2} c_{2}=0, \\
& 3 c_{1}+3 a_{2} c_{1}+6 a_{1} c_{2}=0,12 c_{2}+6 a_{2} c_{2}=0 .
\end{aligned}
$$

With the aid of Mathematica we find two sets of solutions:

$$
\begin{aligned}
& a_{0}=\frac{1}{2}\left(-d-b_{1}^{2}\right), \quad b_{0}=\frac{c_{1}}{2 b_{1}}, \quad a_{2}=-1 \\
& \beta=\frac{1}{2}\left(d+3 b_{1}^{2}\right), \quad a_{1}=b_{2}=c_{2}=0 \\
& a_{0}=-\frac{1}{3}(4 d+\beta), \quad b_{0}=\frac{1}{6}\left(-4 d-4 \beta \pm 3 c_{2}\right) \\
& a_{2}=-2, \quad b_{2}= \pm 1, \quad a_{1}=b_{1}=c_{1}=0 .
\end{aligned}
$$

Since $d$ is arbitrary in (12) and (13), according to (7)-(9), we find six types of travelling wave solutions: 

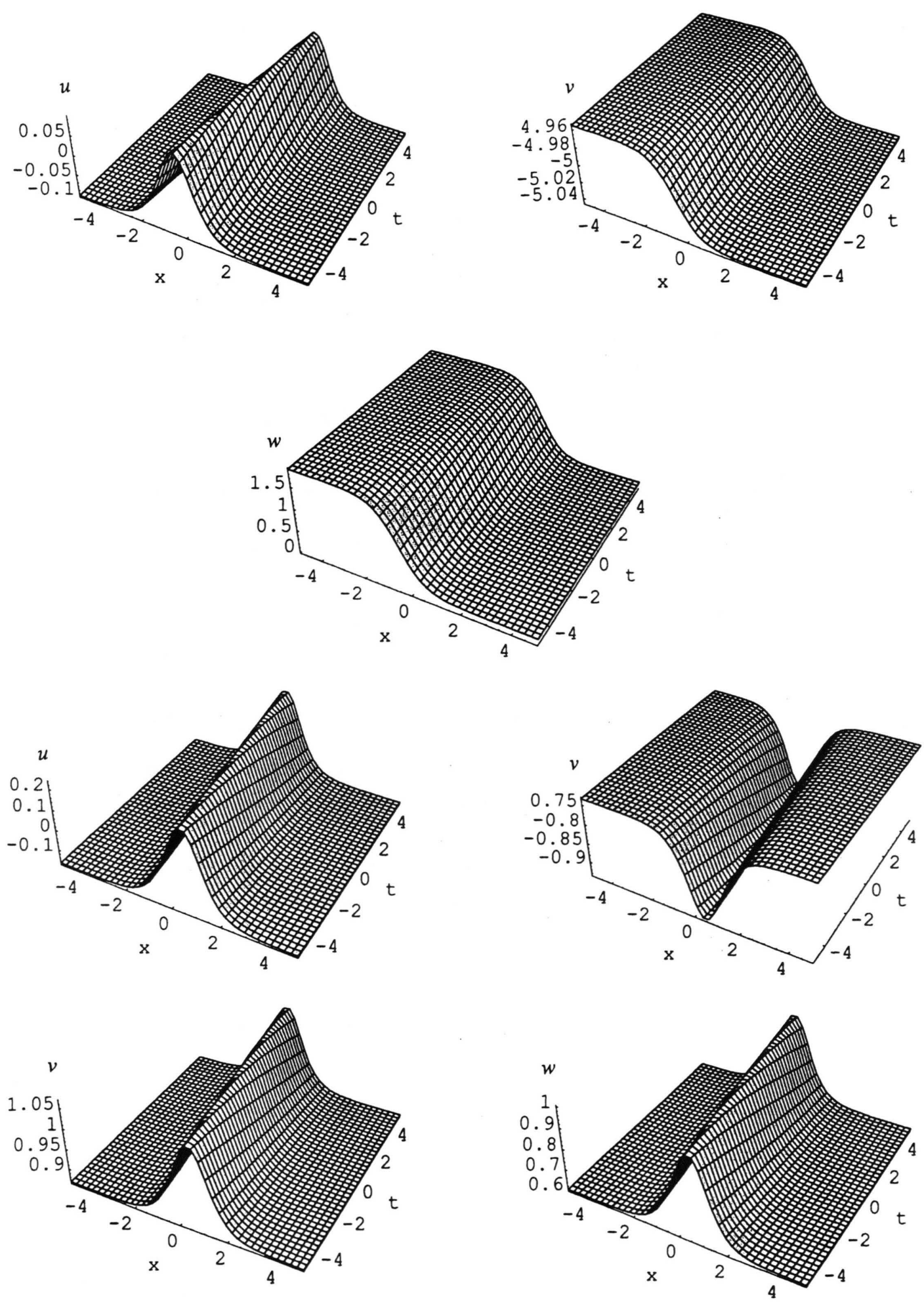

Fig. 1. Plots of $u, v$ and $w$ in the case $d<0$. 
E. Fan - Generalized Hirota-Satsuma Coupled KdV Systems
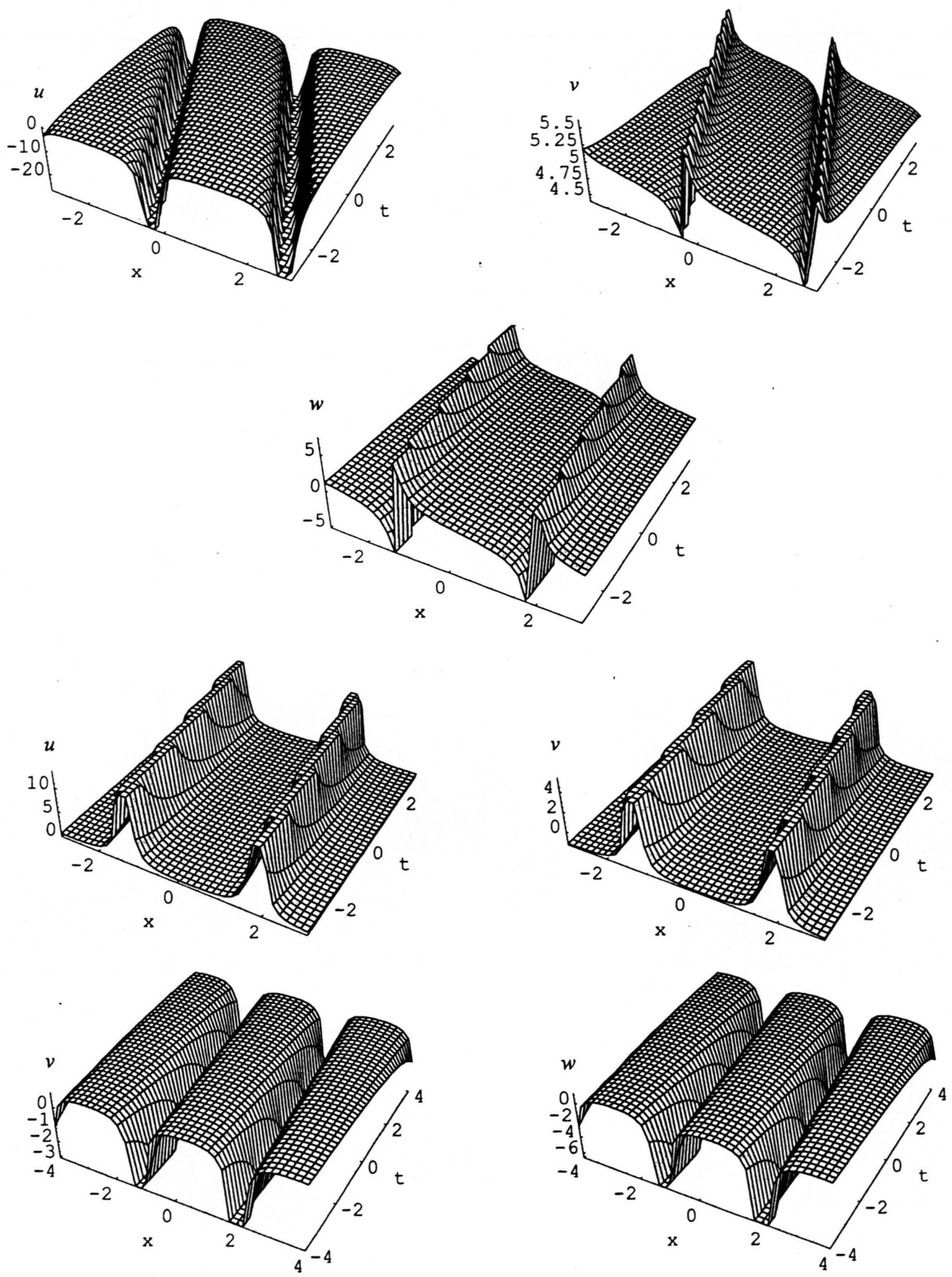

Fig. 2. Plots of $u, v$ and $w$ in the case $d>0$. 


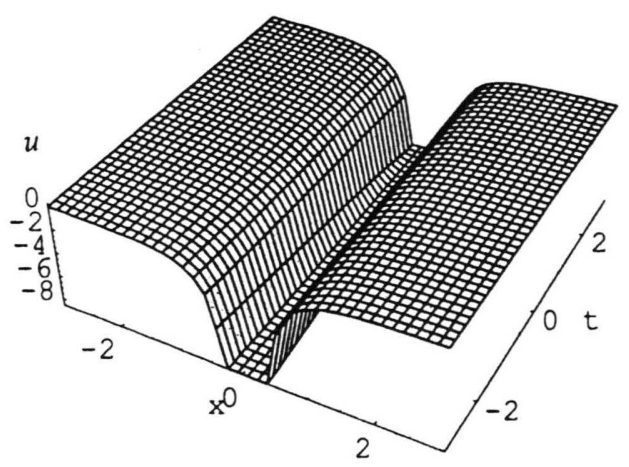

E. Fan - Generalized Hirota-Satsuma Coupled KdV Systems

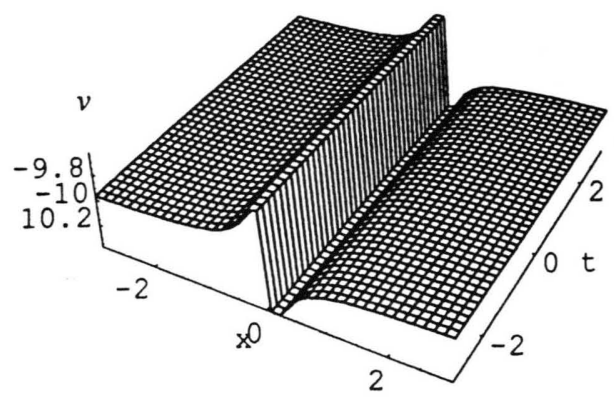

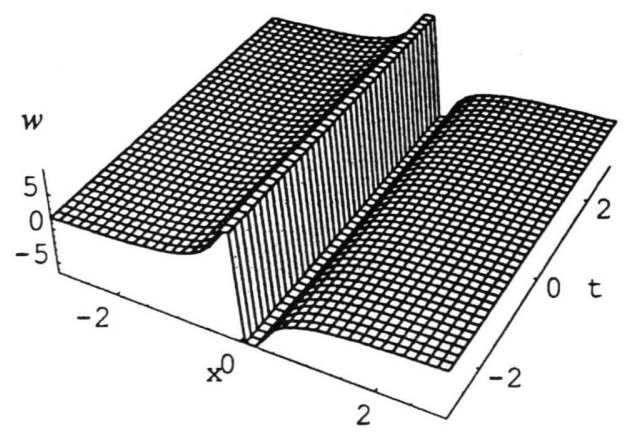
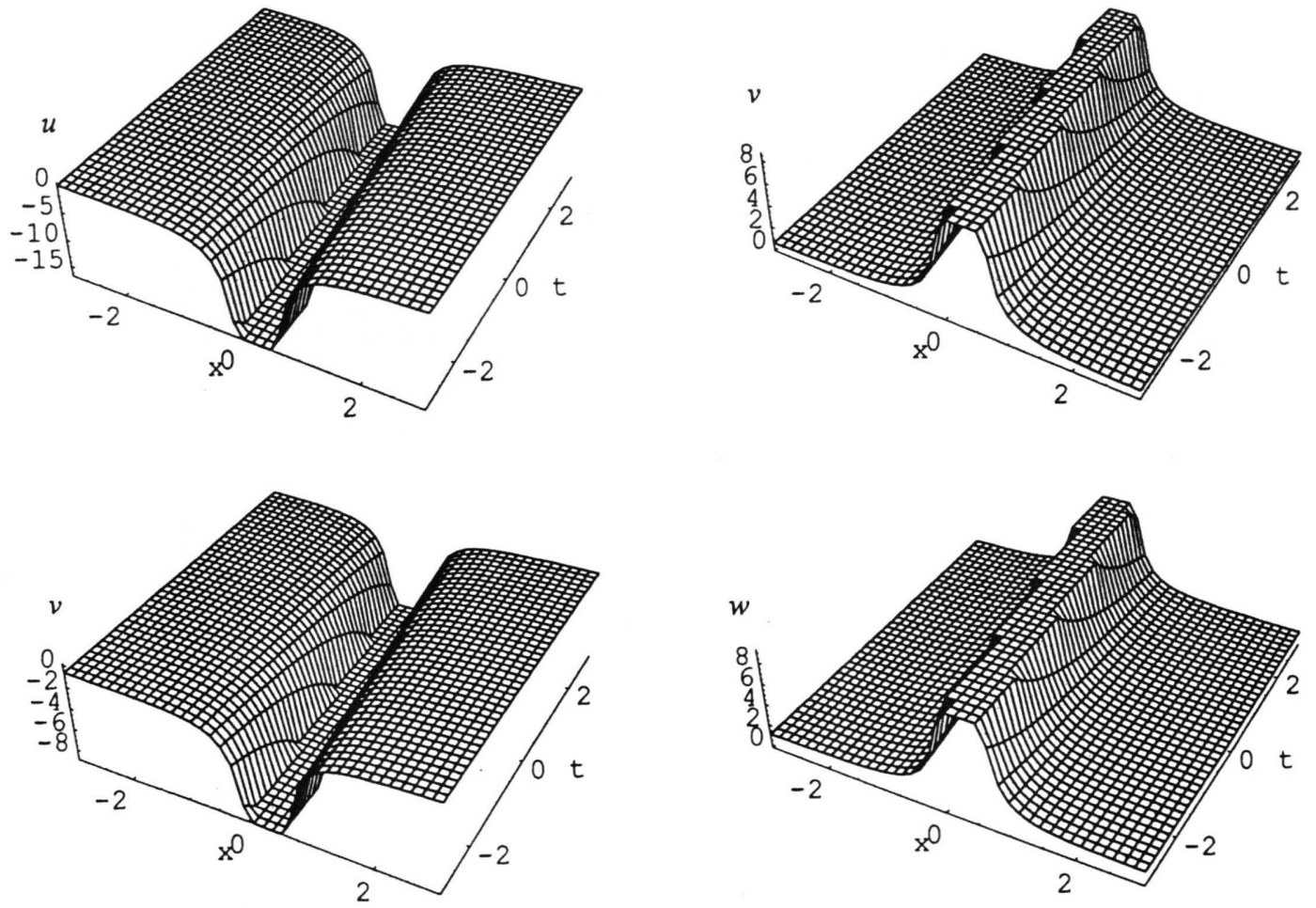

Fig. 3. Plots of $u, v$ and $w$ in the case $d=0$. 
As $d<0$ :

$$
\begin{aligned}
& u=-\frac{1}{2}\left(d+b_{1}^{2}\right)+d T^{2}, \quad v=\frac{c_{1}}{2 b_{1}}-b_{1} \sqrt{-d} T, \\
& w=c_{0}-c_{1} \sqrt{-d} T, \quad \xi=x+\frac{1}{2}\left(d+3 b_{1}^{2}\right) t . \\
& u=-\frac{1}{3}(4 d+\beta)+2 d T^{2}, \\
& v=\frac{1}{6}\left(-4 d-4 \beta \pm 3 c_{2}\right) \mp d T^{2}, \\
& w=c_{0}-c_{2} d T^{2}, \quad \xi=x+\beta t .
\end{aligned}
$$

It is seen that the solutions obtained in this case are soliton solutions. Their physical relevance is clear to us. $u$ in (14) is a bell-type wave and $v, w$ are kink-type waves, while $u, v, w$ in (15) are all bell-type wave (see Figure 1).

As $d>0$ :

$$
\begin{aligned}
& u=-\frac{1}{2}\left(d+b_{1}^{2}\right)-d S^{2}, \\
& v=\frac{c_{1}}{2 b_{1}}-b_{1} \sqrt{d} S, \quad w=c_{0}+c_{1} \sqrt{-d} S, \\
& \xi=x+\frac{1}{2}\left(d+3 b_{1}^{2}\right) t . \\
& u=-\frac{1}{3}(4 d+\beta)-2 d S^{2}, \\
& v=\frac{1}{6}\left(-4 d-4 \beta \pm 3 c_{2}\right) \pm d S^{2}, \\
& w=c_{0}+c_{2}+d S^{2}, \quad \xi=x+\beta t .
\end{aligned}
$$

These solutions are triangle-type periodical solutions (see Fig. 2), which develop a singularity at a finite point, i.e. for any fixed $t=t_{0}$ there exist an $x_{0}$ at which these solutions blow up. There is much current interest in the formation of so called "hot-spots" or "blow-ups" of solutions [8-10]. It appears that the singular solutions will model these physical phenomena.

As $d=0$ :

$$
\begin{aligned}
& u=-\frac{1}{2} b_{1}^{2}-\frac{1}{\xi^{2}}, \quad v=\frac{c_{1}}{2 b_{1}}-\frac{b_{1}}{\xi}, \\
& w=c_{0}-\frac{c_{1}}{\xi}, \quad \xi=x+\frac{3}{2} b_{1}^{2} t . \\
& u=-\frac{1}{3} \beta-\frac{2}{\xi^{2}}, \quad v=\frac{1}{6}\left(-4 \beta \pm 3 c_{2}\right) \pm \frac{1}{\xi^{2}}, \\
& w=c_{0}+\frac{c_{2}}{\xi^{2}}, \quad \xi=x+\beta t .
\end{aligned}
$$

These solutions are rational-type solutions (see Fig. 3), which may be helpful to explain certain physical phe- nomena. Because a rational solution is a disjoint union of manifolds, particle systems describing the motion of a pole of rational solutions for a $\mathrm{KdV}$ equation were analyzed in [11-14].

Remark. Taking $c_{0}=c_{1}=c_{2}=0$ and making the transformation $x \rightarrow \sqrt{2} x, t \rightarrow \sqrt{2} t$, the above solutions reduce to solutions of the Hirota-Satsuma equation (3). It is clear that such solutions are different from those in [3, 4], since the $u, v$ obtained by Hirota-Satsuma $[1,2]$ were

$$
\begin{aligned}
& u=2\left(\ln \left(1+\frac{1}{8 k^{4}} e^{2 \eta}\right)\right)_{x x}, \\
& v=\frac{e^{\eta}}{1+\frac{1}{8 k^{4}} e^{2 \eta}} \cdot \eta=k x-k^{3} t+\eta_{0},
\end{aligned}
$$

while the $u, v$ found by Tam-Ma-Hu [3] were

$$
\begin{aligned}
& u=2\left(\ln \left(1+e^{\eta}+\frac{1}{32}\left(4+k^{4}\right) e^{2 \eta}\right)\right)_{x x}, \\
& v=1+\frac{k^{4} e^{\eta}}{2\left[1+e^{\eta}+\frac{1}{32}\left(4+k^{4}\right) e^{2 \eta}\right]} .
\end{aligned}
$$

In the following we look for travelling wave solutions of the system (2). We make the transformation $u(x, t)=$ $U(\xi), v(x, t)=V(\xi), w(x, t)=W(\xi), \xi=x+\beta t$ and change the system (2) into the form

$$
\begin{aligned}
& \beta U^{\prime}=\frac{1}{2} U^{\prime \prime \prime}-3 U U^{\prime}+3(V W)^{\prime}, \\
& \beta V^{\prime}=-V^{\prime \prime \prime}+3 U V^{\prime}, \\
& \beta W^{\prime}=-W^{\prime \prime \prime}+3 U V^{\prime} .
\end{aligned}
$$

Balancing the highest order linear term with nonlinear terms in (16) admits the ansatz

$$
\begin{gathered}
u=a_{0}+a_{1} \varphi+a_{2} \varphi^{2}, \\
v=b_{0}+b_{1} \varphi+b_{2} \varphi^{2}, \\
w=c_{0}+c_{1} \varphi+c_{2} \varphi^{2},
\end{gathered}
$$

Substituting (17) into (16) yields an algebraic system of $a_{i}, b_{i}, c_{i},(i=0,1,2), d$ and $\beta$ :

$-d^{2} a_{1}+d \beta a_{1}+3 d a_{0} a_{1}-3 d b_{1} c_{0}-3 d b_{0} c_{1}=0$,

$3 d a_{1}^{2}-8 d^{2} a_{2}+2 d \beta a_{2}+6 d a_{0} a_{2}-6 d b_{2} c_{0}$

$-6 d b_{1} c_{1}-6 d b_{0} c_{2}=0$,

$-4 d a_{1}+\beta a_{1}+3 a_{0} a_{1}+9 a_{1} a_{2}-3 b_{1} c_{0}-3 b_{0} c_{1}$

$-9 d b_{2} c_{1}-9 d b_{1} c_{2}=0$,

$-3 a_{1}^{2}-20 d a_{2}+2 \beta a_{2}+6 a_{0} a_{2}+6 d a_{2}^{2}-6 b_{2} c_{0}$

$-6 b_{1} c_{1}-6 b_{0} c_{2}-12 d b_{2} c_{2}=0$,

$-3 a_{1}+9 a_{1} a_{2}-9 b_{2} c_{1}-9 b_{1} c_{2}=0$, 
$-12 a_{2}+6 a_{2}^{2}-12 b_{2} c_{2}=0$,

$2 d^{2} b_{1}+d \beta b_{1}-3 d a_{0} b_{1}=0$,

$-3 d a_{1} b_{1}+16 d^{2} b_{2}+2 d \beta b_{2}-6 d a_{0} b_{2}=0$,

$-3 a_{1} b_{1}+40 d b_{2}+2 \beta b_{2}-6 a_{0} b_{2}-6 d a_{2} b_{2}=0$,

$6 b_{1}-3 a_{2} b_{1}-6 a_{1} b_{2}=0,24 b_{2}-6 a_{2} b_{2}=0$,

$2 d^{2} c_{1}+d \beta c_{1}-3 d a_{0} c_{1}=0$,

$-3 d a_{1} c_{1}+16 d^{2} c_{2}+2 d \beta c_{2}-6 d a_{0} c_{2}=0$,

$8 d c_{1}+\beta c_{1}-3 a_{0} c_{1}-3 d a_{2} c_{1}-6 d a_{1} c_{2}=0$,

$-3 a_{1} c_{1}+40 d c_{2}+2 \beta c_{2}-6 a_{0} c_{2}-6 d a_{2} c_{2}=0$,

$6 c_{1}-3 a_{2} c_{1}-6 a_{1} c_{2}=0,24 c_{2}-6 a_{2} c_{2}=0$,

which has two sets of solutions:

$$
\begin{aligned}
& a_{0}=\frac{1}{3}(8 d+\beta), \quad a_{1}=b_{1}=c_{1}=0, \quad a_{2}=4, \\
& b_{0}=\frac{4\left(4 d c_{2}+2 \beta c_{2}-3 c_{2}\right)}{3 c_{2}^{2}}, \quad b_{2}=\frac{4}{c_{2}} . \\
& a_{0}=\frac{1}{4}\left(4 d+b_{1} c_{1}\right), \quad a_{1}=b_{2}=c_{2}=0, \quad a_{2}=2, \\
& b_{0}=-\frac{b_{1} c_{0}}{c_{1}}, \quad \beta=\frac{1}{4}\left(4 d+3 b_{1} c_{1}\right) .
\end{aligned}
$$

Since $d$ is arbitrary, we also get six travelling wave solutions for system (2):

As $d<0$

$$
\begin{aligned}
& u=\frac{1}{3}(8 d+\beta)-4 d T^{2}, \\
& v=\frac{4\left(4 d c_{2}+2 \beta c_{2}-3 c_{0}\right)}{3 c_{2}^{2}}-\frac{4 d}{c_{2}} T^{2}, \\
& w=c_{0}-c_{1} d T^{2}, \quad \xi=x+\beta t \\
& u=\frac{1}{4}\left(4 d+b_{1} c_{1}\right)-2 d T^{2} \\
& v=-\frac{b_{1} c_{0}}{c_{1}}-b_{1} \sqrt{-d} T \\
& w=c_{0}-c_{1} \sqrt{-d} T, \quad \xi=x+\frac{1}{4}\left(4 d+3 b_{1} c_{1}\right) t
\end{aligned}
$$

[1] J. Satsuma and R. Hirota, J. Phys. Soc. Japan 51, 332 (1982).

[2] R. Hirota and J. Satsuma, Phys. Lett. A 85, 407 (1981).

[3] H. W. Tam, W. X. Ma, and X. B. Hu, J. Phys. Soc. Japan 69, 45 (2000).

[4] Y. T. Wu, X. G. Geng, X. B. Hu, and S. M. Zhu, Phys. Lett. A 255, 259 (1999).

[5] W. Malfliet, Amer. J. Phys. 60, 650 (1992).

[6] E. J. Parkes and B. R. Duffy, Computer Phys. Commun. 98, 288 (1996).

[7] Y. T. Gao and B. Tian, Z. Naturforsch. 52A, 372 (1997).
As $d>0$

$$
\begin{aligned}
& u=\frac{1}{3}(8 d+\beta)+4 d S^{2}, \\
& v=\frac{4\left(4 d c_{2}+2 \beta c_{2}-3 c_{0}\right)}{3 c_{2}^{2}}+\frac{4 d}{c_{2}} S^{2}, \\
& w=c_{0}+c_{1} d S^{2}, \quad \xi=x+\beta t . \\
& u=\frac{1}{4}\left(4 d+b_{1} c_{1}\right)+2 d S^{2}, \\
& v=-\frac{b_{1} c_{0}}{c_{1}}+b_{1} \sqrt{d} S, \quad w=c_{0}+c_{1} \sqrt{d} S, \\
& \xi=x+\frac{1}{4}\left(4 d+3 b_{1} c_{1}\right) t .
\end{aligned}
$$

As $d=0$

$$
\begin{aligned}
& u=\frac{1}{3} \beta+\frac{4}{\xi^{2}}, \quad v=\frac{4\left(2 \beta c_{2}-3 c_{0}\right)}{3 c_{2}^{2}}+\frac{4}{c_{2} \xi^{2}}, \\
& w=c_{0}+\frac{c_{2}}{\xi^{2}}, \quad \xi=x+\beta t . \\
& u=\frac{1}{4} b_{1} c_{1}+\frac{2}{\xi^{2}}, \quad v=-\frac{b_{1} c_{0}}{c_{1}}-\frac{b_{1}}{\xi}, \\
& w=c_{0}-\frac{c_{1}}{\xi}, \quad \xi=x+\frac{3}{4} b_{1} c_{1} t .
\end{aligned}
$$

Since the properties of these solutions are similar to those of system (1), we omit their plots and discussion here.

\section{Acknowledgements}

I would like to express my sincere thanks to a referee for his very help advice and suggestions. I also am grateful to Prof. Gu Chaohao, Prof. Hu Hesheng, and Prof. Zhou Zixiang for their enthusiastic guidance and help. This work is supported by the Chinese Basic Research Plan "Mathematics Mechanization and a Platform for Automated Reasoning", the Postdoctoral Science Foundation of China and the Shanghai Postdoctoral Science Foundation of China.
[8] N. F. Smyth, J. Aust. Math. Soc. Ser B 33403 (1992).

[9] P. A. Clarkson and E. L. Manfield, Physica D 70, 250 (1993).

[10] N. A. Kudryashov and D. Zargayan, J. Phys. A 29, 8067 (1996).

[11] M.Adler and J. Moser, Commun. Math. Phys. 19, 1 (1978).

[12] A. Nakamura and R. Hirota, J. Phys. Soc. Japan. 54, 491 (1985).

[13] R. L. Sachs, Physica D 30, 1 (1988).

[14] E. G. Fan and H. Q. Zhang, Phys. Lett. A 245, 389 (1998). 